\title{
Residual Entropy and Low Temperature Pseudo-Transition for One-Dimensional Models
}

\author{
O. RoJAs* \\ Departamento de Física, Universidade Federal de Lavras, 37200-900, Lavras-MG, Brazil
}

\begin{abstract}
Here we report an intrinsic relationship between the zero temperature phase boundary residual entropy and pseudo-transition. Usually, the residual entropy increases at the phase boundary, which means the system gains accessible states in the phase boundary compared to its adjacent states, although it is not always the case. Therefore, we propose the following statement at zero temperature. If the residual entropy is continuous at least from the one-sided limit, then the analytical free energy exhibits a pseudo-transition at low temperature. For illustrative purpose, our argument is applied to a frustrated coupled double tetrahedral Ising-Heisenberg chain to show the pseudo-transitions behaviors due to the phase boundary residual entropy continuity.
\end{abstract}

DOI: 10.12693/APhysPolA.137.933

PACS/topics: residual entropy, pseudo-transition, quasi-phase

\section{Introduction}

In 1950, van Hove [1] verified the absence of a phase transition for a short-range interaction and uniform onedimensional system. Using the transfer matrix technique, we can get the free energy, which must be an analytical function, thereby establishing a theorem to prove that one-dimensional models with short-range coupling do not exhibit any phase transitions. Later, Cuesta and Sanchez [2] generalized the theorem of non-existence of phase transition at finite temperatures by including an external field and considering point-like particles. It broadens the non-existence theorem, but it is far from being a sufficiently general theorem.

On the other hand, the term "pseudotransition" was introduced by Timonin [3] in 2011 while studying a spin ice in a field, and refers to a sudden change in the first derivative of the free energy, while a sharp vigorous peak appears in the second derivative of the free energy, although there are no discontinuity or divergence. The pseudotransition does not violate the Perron-Frobenius theorem [4], because the free energy is analytic.

To illustrate this behavior better, let us consider a decorated model $[5,6]$ that can be mapped in a simple spin-1/2 Ising-like model with Hamiltonian

$$
H=-\sum_{i=1}^{N}\left[K_{0}+K s_{i} s_{i+1}+\frac{1}{2} B\left(s_{i}+s_{i+1}\right)\right]
$$

where $K_{0}, K$, and $B$ are effective parameters which in general could depend on temperature, and $N$ denotes unit cells. The corresponding transfer matrix is expressed by

$$
\boldsymbol{V}=\left[\begin{array}{cc}
w_{1} & w_{0} \\
w_{0} & w_{-1}
\end{array}\right]
$$

*e-mail: ors@ufla.br as discussed in [5]. Each element of transfer matrix is given by

$$
w_{n}=\sum_{k=0} g_{n, k} \exp \left(-\beta \varepsilon_{n, k}\right) .
$$

Here, $\varepsilon_{n, k}$ represent the energy spectra, where $k=\{0,1, \ldots\}$ for each $n$, while $g_{n, k}$ denotes its corresponding degeneracy $g_{n, k}=\{1,2,3, \ldots\}$. Coefficient $\beta=1 /\left(k_{\mathrm{B}} T\right)$, where $k_{\mathrm{B}}$ is the Boltzmann constant, and $T$ is the absolute temperature.

Consequently, the free energy in the thermodynamic limit $(N \rightarrow \infty)$ becomes

$$
f=-\frac{1}{\beta} \ln \left(\frac{1}{2}\left(w_{1}+w_{-1}\right)+\frac{1}{2} \sqrt{\left(w_{1}-w_{-1}\right)^{2}+4 w_{0}^{2}}\right) .
$$

When $w_{0}=0$, Eq. (3) indicates the presence of a genuine finite temperature phase transition at $w_{1}=w_{-1}$.

The organization of this report is as follows. In Sect. 2 the critical residual entropy is presented. In Sect. 3, we study for a double tetrahedral Ising-Heisenberg chain. Finally, in Sect. 4, we present our conclusions.

\section{Phase boundary residual entropy}

Let us assume that the energies $\varepsilon_{1,0}$ and $\varepsilon_{-1,0}$ are the lowest energies for $n=1$ and $n=-1$, respectively, and depend on control parameter $x$, e.g. magnetic field. Thus, the energies $\varepsilon_{1,0}(x)$ and $\varepsilon_{-1,0}(x)$, in the critical point become $\varepsilon_{1,0}\left(x_{c}\right)=\varepsilon_{-1,0}\left(x_{c}\right)=\varepsilon_{c}$, with its corresponding critical degeneracies at phase boundary as $g_{1,0}$ and $g_{-1,0}$. The lowest energy, for $n=0$, satisfies $\varepsilon_{0,0}\left(x_{c}\right)>\varepsilon_{c}$, when $\bar{w}_{0} \rightarrow 0$. Thus, the free energy around the phase boundary becomes

$$
\begin{aligned}
f=- & \frac{1}{\beta} \ln \left(\frac{1}{2}\left(g_{1,0}+g_{-1,0}+\left|g_{1,0}-g_{-1,0}\right|\right) \mathrm{e}^{-\beta \varepsilon_{c}}\right)= \\
& \varepsilon_{c}-\frac{1}{\beta} \ln \left(\max \left(g_{1,0}, g_{-1,0}\right)\right) .
\end{aligned}
$$

Next, we can obtain the corresponding critical residual entropy, 


$$
\mathcal{S}_{c}=\ln \left(\max \left(g_{1,0}, g_{-1,0}\right)\right),
$$

and from now on, we will consider the entropy in units of $k_{\mathrm{B}}$. The critical degeneracy per unit cell results in $G_{c}=\max \left(g_{1,0}, g_{-1,0}\right)$.

It is worth mentioning that, according to the third law of thermodynamics or Nernst's postulate: The entropy goes to a constant when $T \rightarrow 0$, and must be independent of any thermodynamic variables such as $x$.

First, let us assume $g_{1,0}=g_{-1,0}$, then the residual entropy is $\lim _{x \rightarrow x^{ \pm}} \mathcal{S}(x)=\mathcal{S}\left(x_{c}\right)=\mathcal{S}_{c}$. When $g_{-1,0}<g_{1,0}$, we have $\lim _{x \rightarrow x_{c}^{-}} \mathcal{S}(x)<\lim _{x \rightarrow x_{c}^{+}} \mathcal{S}(x)=\mathcal{S}_{c}$. Second, we have, $\mathcal{S}_{c}=\max \left(g_{1,0}, g_{-1,0}\right)$, then $\lim _{x \rightarrow x_{c}^{ \pm}} \mathcal{S}(x)<\mathcal{S}\left(x_{c}\right)=\mathcal{S}_{c}$.

In summary, if the residual entropy is continuous at least from the one-sided limit, then analytic free energy exhibits a pseudotransition at low temperature.

Therefore, the pseudocritical temperature [5] can be found using the following relation:

$$
w_{1}\left(x_{p}, T_{p}\right)=w_{-1}\left(x_{p}, T_{p}\right),
$$

which corresponds to a pseudocritical point at $x_{p}$ and $T_{p}$.

\section{Coupled tetrahedral Ising-Heisenberg chain}

The Heisenberg version of the coupled tetrahedral Heisenberg chain was considered in Ref. [7, 8]. Recently, the Ising-Heisenberg tetrahedral chain has been discussed in $[9,10]$. Here we consider a slightly different model (see Fig. 1), and we focus on the pseudotransition property. Thus, the Hamiltonian of the model is

$$
\begin{aligned}
H & =-\sum_{i=1}^{N}\left\{J\left(\boldsymbol{S}_{a, i}, \boldsymbol{S}_{b, i}\right)_{z}+J\left(\boldsymbol{S}_{b, i}, \boldsymbol{S}_{c, i}\right)_{z}\right. \\
& +J\left(\boldsymbol{S}_{c, i}, \boldsymbol{S}_{a, i}\right)_{z}+\frac{h}{2}\left(\sigma_{i}+\sigma_{i+1}\right) \\
& \left.+\left(S_{a, i}^{z}+S_{b, i}^{z}+S_{c, i}^{z}\right)\left[h+J_{0}\left(\sigma_{i}+\sigma_{i+1}\right)\right]\right\} .
\end{aligned}
$$

Here, $J\left(\mathbf{S}_{a, i}, \mathbf{S}_{b, i}\right)_{z} \equiv J S_{a, i}^{x} S_{b, i}^{x}+J S_{a, i}^{y} S_{b, i}^{y}+J_{z} S_{a, i}^{z} S_{b, i}^{z}$, where $S_{a, i}^{\alpha}$ denotes the Heisenberg spin-1/2 with $\alpha=\{x, y, z\}$, while $\sigma_{i}$ denotes the Ising spin $\left(\sigma_{i}= \pm \frac{1}{2}\right)$. Analogous definitions are used for sites $b$ and $c$. It is noteworthy that $J$ and $J_{z}$ correspond to the exchange interaction between the Heisenberg spin, $J_{0}$ stands for the Ising-Heisenberg coupling, and $h=g \mu_{\mathrm{B}} B / k_{\mathrm{B}}$ denotes magnetic field along the $z$-axis. Then all quantities are in units of $k_{\mathrm{B}}$, and consequently, the amounts mentioned above must be measured in kelvin.

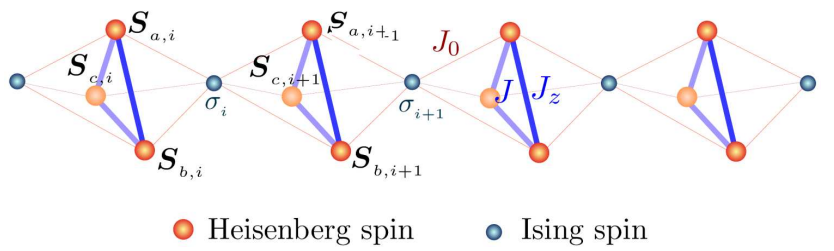

Fig. 1. Schematic representation of coupled tetrahedral Ising-Heisenberg chain.
To analyse the phase diagram at zero temperature, we will discuss some relevant states below.

First, we report the ground state of the saturated phase (SA) as

$$
\begin{aligned}
& |S A\rangle=\prod_{i=1}^{N}\left|\begin{array}{l}
+ \\
+
\end{array}\right\rangle_{i}|+\rangle_{i}, \\
& \text { with } m_{I}=\frac{1}{2}, m_{H}=\frac{1}{2} \text { and } m_{t}=2 .
\end{aligned}
$$

Similarly, the ground state for ferrimagnetic (FI) phase can be expressed as

$$
\begin{aligned}
& \left.|\mathrm{FI}\rangle=\left.\prod_{i=1}^{N}\right|_{+} ^{+}\right\rangle_{i}|-\rangle_{i}, \\
& \text { with } m_{I}=-\frac{1}{2}, m_{H}=\frac{1}{2} \text {, and } m_{t}=1 .
\end{aligned}
$$

The next phase we consider is a frustrated phase, given by

$$
\begin{aligned}
& \left|\mathrm{FR}_{1}\right\rangle=\prod_{i=1}^{N}\left|\frac{1}{2},-\frac{1}{2}\right\rangle_{i}|+\rangle_{i}, \\
& \text { with } m_{I}=\frac{1}{2}, m_{H}=-\frac{1}{6} \text { and } m_{t}=0,
\end{aligned}
$$
where

$$
\begin{aligned}
& \left|\frac{1}{2},-\frac{1}{2}\right\rangle \equiv \frac{1}{\sqrt{6}}\left(\left|\begin{array}{l}
- \\
\frac{+}{+}
\end{array}\right\rangle-2\left|\begin{array}{l}
- \\
+
\end{array}\right\rangle+\left|\begin{array}{l}
+ \\
-
\end{array}\right\rangle\right) \\
& \text { or }\left|\frac{1}{2},-\frac{1}{2}\right\rangle \equiv \frac{1}{\sqrt{2}}\left(\left|\begin{array}{l}
- \\
+
\end{array}\right\rangle-\left|\begin{array}{l}
+ \\
-
\end{array}\right\rangle\right) .
\end{aligned}
$$

The other frustrated ground state energy is

$$
\begin{aligned}
& \left|\mathrm{FR}_{2}\right\rangle=\prod_{i=1}^{N}\left|\frac{1}{2},+\frac{1}{2}\right\rangle_{i}|+\rangle_{i}, \\
& \text { with } m_{I}=\frac{1}{2}, m_{H}=\frac{1}{6} \text { and } m_{t}=1 .
\end{aligned}
$$
where

$$
\begin{aligned}
& \left|\frac{1}{2}+\frac{1}{2}\right\rangle \equiv \frac{1}{\sqrt{6}}\left(\left|\begin{array}{l}
+ \\
+_{-}
\end{array}\right\rangle-2\left|\begin{array}{l}
+ \\
+
\end{array}\right\rangle+\left|\begin{array}{l}
- \\
+
\end{array}\right\rangle\right) \\
& \text { or }\left|\frac{1}{2},+\frac{1}{2}\right\rangle \equiv \frac{1}{\sqrt{2}}\left(\left|\begin{array}{l}
- \\
+
\end{array}\right\rangle-\left|\begin{array}{l}
+ \\
+
\end{array}\right\rangle\right) .
\end{aligned}
$$

In Fig. 2a, the phase diagram is shown at zero temperature. The CRE between $\mathrm{FR}_{1}$ and $\mathrm{FR}_{2}$ is $\mathcal{S}_{c}=\ln (3+\sqrt{5})$, in units of the Boltzmann constant. In the interface between $\mathrm{FR}_{1}$ and FI we have $\mathcal{S}_{c}=\ln (3)$. In the same way, the CRE between FI and SA is $\mathcal{S}_{c}=\ln (2)$, whereas at the boundary between $\mathrm{SA}$ and $\mathrm{FR}_{2}$ we have $\mathcal{S}_{c}=\ln (3)$. All the above critical residual entropies are discontinuous, indicating the absence of the pseudotransition at finite temperature (see Fig. 2b). Finally, the critical residual entropy in the interface of FI and $\mathrm{FR}_{2}$ described by a red solid line is given by $\mathcal{S}_{c}=\ln (2)$. Therefore, we can affirm that this boundary should lead to a pseudotransition (see Fig. 2b). 

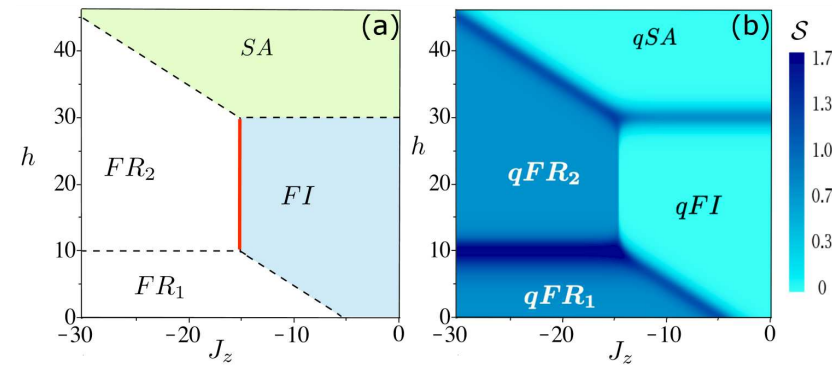

Fig. 2. (a) Zero temperature phase diagram in the plane of $J_{z}-h$, assuming fixed parameters $J=-10$ and $J_{0}=-10$. (b) Entropy density plot for temperature $T=0.6$, assuming the same set of parameters considered in (a). Here the prefix letter "q" is to assign the quasi-phases.
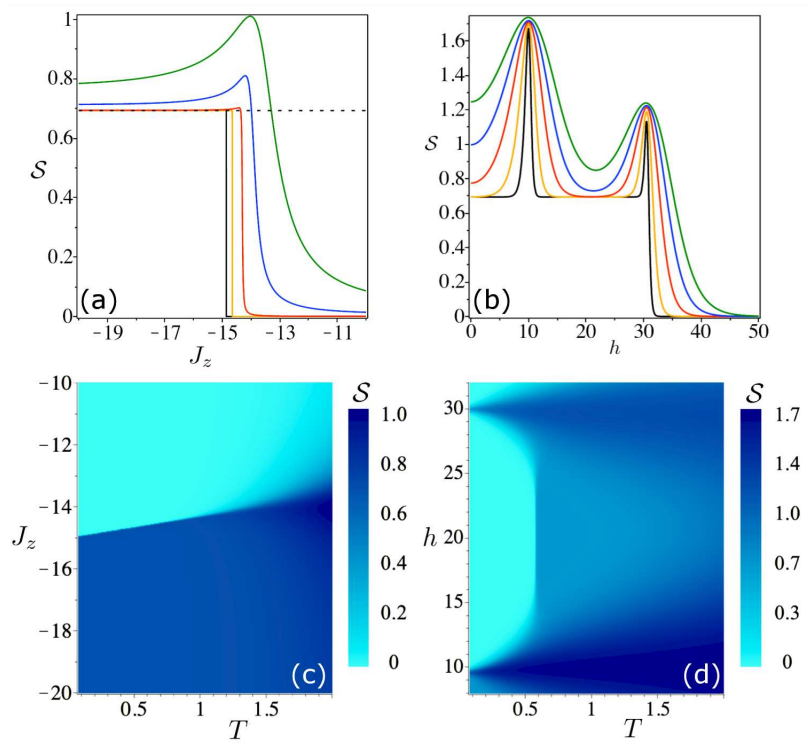

Fig. 3. Entropy as a function of $J_{z}$ assuming fixed $J=-10, J_{0}=-10$, for a range of temperature, $T=\{0.2,0.5,1.0,1.5,2.0\}$ (inner to outer curve): (a) for $h=20$, and (b) for $J_{z}=-20$. Density plot of entropy for fixed $J=-10, J_{0}=-10$ : (c) in the plane $J_{z}-T$ for $h=20$, and (d) in the plane $h-T$ for $J_{z}=-14.6$.

To study the thermodynamics of the present model, the Boltzmann factor $(n=\{-1,0,1\})$ can be expressed as

$$
\begin{aligned}
w_{n} & =2 \exp \left(\frac{\beta}{4}\left(2 n h-J_{z}\right)\right) \\
\times & {\left[\left(\mathrm{e}^{\beta J}+2 \mathrm{e}^{-\frac{1}{2} \beta J}\right) \cosh \left(\beta \frac{J_{0}+h_{z}}{2}\right)\right.} \\
& \left.+\mathrm{e}^{\beta J_{z}} \cosh \left(3 \beta \frac{J_{0}+h_{z}}{2}\right)\right],
\end{aligned}
$$

In Fig. 3a the entropy is shown as a function of $J_{z}$ in the low-temperature region, where we can observe the critical residual entropy between $\mathrm{FR}_{2}$ and $\mathrm{FI}$ as $\mathcal{S}_{c}=\ln (2)$, and this amount remains almost constant up to $T \lesssim 1$. This is because the entropy is continuous from the one-sided limit at phase boundary. In Fig. 3b the entropy is plotted as a function of $h$, where we observe that the residual entropy peaks $\mathcal{S}_{c}=\ln (4)$ and $\mathcal{S}_{c}=\ln (2)$ that occur for $h=10$ and $h=30$, respectively. In Fig. 3c we observe well distinct regions up to $T \lesssim 1$, and for higher temperature the boundary becomes blurry. Similarly, in Fig. 3d there is a sharp boundary for $T \approx 0.6$ and $15 \lesssim h \lesssim 25$. Outside this region thermal excitation destroys any trace of phase transition at zero temperature.

\section{Conclusions}

Usually, the residual entropy increases in the interface where the phase transition occurs. However, there are some peculiar cases where the critical residual entropy is equal to the largest residual entropy of neighboring states, which is given by $\mathcal{S}_{c}=\ln \left(\max \left(g_{1,0}, g_{-1,0}\right)\right)$. We can apply this condition at zero temperature, and searching for the continuity of entropy would be an easier task when compared to the study of full thermodynamic quantities. To show this property, we have considered a double tetrahedral Ising-Heisenberg chain.

\section{Acknowledgments}

This work was supported by the Brazilian financial agencies CNPq and FAPEMIG.

\section{References}

[1] L. van Hove, Physica 16, 137 (1950).

[2] J.A. Cuesta, A. Sanchez, J. Statist. Phys. 115, 869 (2003).

[3] P.N. Timonin, J. Exp. Theor. Phys. 113, 251 (2011).

[4] F. Ninio, J. Phys. A Math. Gen. 9, 1281 (1976).

[5] S.M. de Souza, O. Rojas, Solid State Commun. 269, 131 (2017).

[6] L. Galisova, J. Strecka, Phys. Rev. E 91, 0222134 (2015).

[7] M. Mambrini, J. Trébosc, F. Mila, Phys. Rev. B 59 13806 (1999).

[8] O. Rojas, F.C. Alcaraz, Phys. Rev. B 67, 174401 (2003).

[9] V. Ohanyan, Condens. Matter Phys. 12, 343 (2009).

[10] D. Antonosyan, S. Bellucci, V. Ohanyan, Phys. Rev. B 79, 014432 (2009). 